\title{
Is levetiracetam neuroprotective in neonatal rats with hypoxic ischemic brain injury?
}

\author{
Celik Y ${ }^{1}$, Resitoglu B ${ }^{2}$, Komur $\mathrm{M}^{3}$, Polat $\mathrm{A}^{4}$, Arslankoylu AE $\mathrm{A}^{5}$, Okuyaz $\mathrm{C}^{3}$, Erdogan $\mathrm{S}^{6}$, Beydagi $\mathrm{H}^{2}$
}

Mersin University Faculty of Medicine, Department of Pediatrics, Division of Neonatology 33343, Mersin, Turkey.drycelik@yahoo.com

\begin{abstract}
BACKGROUND: The aim of this study was to determine if levetiracetam (LEV) is neuroprotective in neonatal rats with hypoxic-ischemic brain injury (HIBI).

METHODS: The study included 7-d-old male Wistar rats that were randomly divided into the LEV400, LEV800, control, and sham groups. All the rats, except those in the sham group, underwent ligation of the carotid artery and were then kept in a hypoxic chamber containing $8 \%$ oxygen for $2 \mathrm{~h}$. At the end of the hypoxic period the rats in the control group were administered saline solution $0.5 \mathrm{~mL}$, the rats in the LEV400 group were administered LEV $400 \mathrm{mg} \cdot \mathrm{kg}^{-1}$, and rats in the LEV800 group were administered LEV $800 \mathrm{mg} \cdot \mathrm{kg}^{-1}$ via the intraperitoneal route. The terminal deoxynucleotidyl transferase-mediated deoxyuridine triphosphate nick-end labeling (TUNEL) method was used to evaluate neuronal apoptosis in the rats. The Morris Water Maze (MWM) test was performed at age 14 weeks in order to evaluate cognitive function.

RESULTS: The number of apoptotic neurons in the right hemispheres was significantly lower in the sham, LEV400, and LEV800 groups than in the control group $(p<0.001, p<0.001$, and $p<0.001$, respectively). In addition, the number of apoptotic neurons in the right hemispheres was significantly lower in the LEV800 group than in the LEV400 group $(p=0.001)$. Platform finding time (PFT) during MWM testing was significantly shorter in the sham and LEV800 groups on $d 4$ than on $d 1(p=0.001$ and $p=0.006$, respectively); however, PFT did not significantly change between $d 1$ and $d 4$ in the control or LEV400 groups ( $p=0.91$ and $p=0.096$, respectively). CONCLUSION: Based on the present findings, LEV exhibited a dose-dependent neuroprotective effect in neonatal rats with HIBI (Ref. 27). Text in PDF www.elis.sk.

KEY WORDS: hypoxic ischemic encephalopathy, levetiracetam, new-born.
\end{abstract}

\section{Introduction}

Hypoxic-ischemic encephalopathy (HIE) is the leading cause of neonatal disability and mortality (1). Approximately 20-30\% of new-borns with HIE die after birth and almost $50 \%$ of those that survive do so with a significant disability, such as cerebral palsy and mental retardation $(2,3)$. Currently, therapeutic hypothermia (TH) is routinely used to treat moderate-severe HIE (4). Unfortunately, TH is not adequately effective in all new-borns with HIE, as a very high percentage of new-borns with HIE develop disabilities despite of the use of TH (5). As such, new treatment methods are required for HIE that are used alone or in combination with TH (4).

${ }^{1}$ Mersin University Faculty of Medicine, Department of Pediatrics, Division of Neonatology, Mersin, Turkey, ${ }^{2}$ Mersin University Faculty of Medicine, Department of Physiology, Mersin, Turkey, ${ }^{3}$ Mersin University Faculty of Medicine, Department of Pediatric Neurology, Mersin, Turkey, ${ }^{4}$ Mersin University Faculty of Medicine, Department of Pathology, Mersin, Turkey, ${ }^{5}$ Mersin University Faculty of Medicine, Department of Pediatrics, Mersin, Turkey, and ${ }^{6}$ Mersin University Faculty of Medicine, Department of Biostatistics, Mersin, Turkey

Address for correspondence: Y. Celik, Mersin University Faculty of Medicine, Department of Pediatrics, Division of Neonatology 33343, Mersin, Turkey.

Phone: +90.324 .2410000 .2618 , Fax: +90.324 .2410098$
New-borns with HIE frequently suffer from seizures (6) and conventional antiepileptic drugs (AEDs), such as: phenobarbital, phenytoin, and benzodiazepines, are used to treat such seizures (7). Some clinicians think that conventional AEDs can have negative effects on the development of the nervous system (7) based on findings indicating that conventional AEDs cause neuronal apoptosis and neurodegeneration in the immature brain and impair cognitive functions $(8,9)$. Animal studies on the novel antiepileptic drug levetiracetam (LEV) did not observe such neurodegenerative effects $(10,11)$. Furthermore, LEV administered at doses beyond the therapeutic range did not increase apoptotic neuron numbers (12), LEV was reported to have a positive effect on neurodevelopmental, in contrast to conventional AEDs (7).

Due to its intriguing characteristics, some have begun to use LEV for the treatment of hypoxic-ischemic brain injury (HIBI) and several experimental studies on LEV for the treatment of HIBI have been conducted (13-15). One study reported that LEV significantly decreased apoptotic neuron numbers in a neonatal rat model of HIBI (13). Another study reported that LEV significantly decreased apoptotic neuron numbers and improved cognitive functions in a neonatal rat model of HIBI (14). In contrast to these 2 studies that indicate the positive effects of LEV in a neonatal rat model of HIBI, another study reported that LEV did not have a neuroprotective effect in a neonatal animal model of HIBI (15). 
Based on the current literature, the neuroprotective effect of LEV on HIBI remains unclear. The present study, therefore, aimed to determine if high-dose LEV has a neuroprotective effect in neonatal rats with HIBI.

\section{Methods}

\section{Study design and animals}

This randomized, controlled animal study included neonatal male Wistar rats aged $7 \mathrm{~d}$. The study was conducted at Mersin University, School of Medicine, Experimental Animals Research Laboratory and Physiology Behavior Laboratory, Mersin, Turkey. The Mersin University Animal Experiments Ethics Committee approved the study protocol.

\section{Experimental groups and hypoxia-ischemia model}

The rats $(n=64)$ were randomly divided into 4 equally sized groups: LEV400, LEV800, control, and sham. The neonatal rats received halothane inhalation anaesthesia for $<5 \mathrm{~min}$, and then HIBI was induced according to the Levine-Rice model (16). In all rats, except those in sham group, the right carotid artery was tied with 6.0 silk sutures, followed by exposure to hypoxia for $2 \mathrm{~h}$ in a hypoxia chamber containing $8 \%$ oxygen and $92 \%$ nitrogen, and then administration of treatment according to group:

LEV400 group $(\mathrm{n}=16)$ : The rats received LEV $400 \mathrm{mg} \cdot \mathrm{kg}^{-1}$ dissolved in saline via the intraperitoneal route immediately after hypoxia;

LEV800 group $(\mathrm{n}=16)$ : The rats received LEV $800 \mathrm{mg} \cdot \mathrm{kg}^{-1}$ dissolved in saline via the intraperitoneal route immediately after hypoxia;

Control group $(\mathrm{n}=16)$ : The rats received saline $0.5 \mathrm{~mL}$ via the intraperitoneal route immediately after hypoxia;

Sham group $(\mathrm{n}=16)$ : Following neck dissection, the right carotid artery was located but not tied and hypoxia was not induced.

Post treatment, all of the rats were placed with their mother for a 2-h recovery period. Following the recovery period, 8 rats from each group were euthanized via cervical dislocation and decapitated. Brains were removed for analysis of neuronal apoptosis using the terminal deoxynucleotidyl transferase-mediated deoxyuridine triphosphate nick-end labeling (TUNEL) method. The remaining rats underwent Morris Water Maze (MWM) testing at age 14 weeks.

\section{Histopathological evaluation}

Rat brains were examined by a pathologist blinded to which carotid artery was tied and to the treatment groups. From each euthanized rat, 1-2 samples were obtained from the subthalamic nucleus, hippocampus, and parietal cortex. The TUNEL method (in situ apoptosis detection kit, Biogen, catalog no. S7101) was used to detect neuronal DNA fragmentation.

\section{TUNEL method and evaluation of apoptosis}

Coronal brain sections $5 \mathrm{~mm}$ thick were deparaffinised and treated with alcohol, and then incubated with protein kinase $\mathrm{K}$ at room temperature for $15 \mathrm{~min}$. Next, endogen peroxidase activity was extinguished using $2 \% \mathrm{H}_{2} \mathrm{O}_{2}$, and then the slices were incubated at $37{ }^{\circ} \mathrm{C}$ for $60 \mathrm{~min}$ in a moist chamber with $50 \mu \mathrm{L}$ of TdT buffer. Lastly, streptavidin-biotin-peroxidase complex and diaminobenzidine were used to render the reaction visible. TUNEL-labeled slides were counter stained with $1 \%$ methyl green. Apoptotic neurons in subthalamic nucleus, hippocampus, and parietal cortex samples obtained from both the right and left hemispheres were enumerated. Total TUNEL-positive stained neurons were calculated via light microscopy in 5 high-power fields (5' 400) to evaluate numeric density.

\section{MWM test}

Learning and memory were evaluated via standard MWM testing (17). When the rats were aged 14 weeks, MWM testing for $5 \mathrm{~d}$ was performed. Two d before starting MWM testing, the rats were brought to the behavioural experiments laboratory for habituation. Water at $22{ }^{\circ} \mathrm{C}$ was used to fill a tank $42 \mathrm{~cm}$ deep. The same investigator placed the rats in the tank during all MWM tests. In all groups, MWM testing was performed between 0900 and 1400. The tank was divided into 4 equal quadrants (west, north, east, and south) and its image was transmitted to a computer screen. On d 1-4 of MWM testing a 15-cm diameter platform was placed in the middle of the east quadrant and adjusted to a height of 40 $\mathrm{cm}$, so that it could not be observed from outside the tank. On $\mathrm{d} 1$ of the experiment, all rats were placed in the water once in each quadrant, starting from the west quadrant and proceeding clockwise to the other 3, with their heads turned toward the wall of the water maze. On each successive d (d 2-4) of the experiment, the rats were placed first in the north, east, and then south quadrant.

When the rats were dropped into the water they were expected to find the hidden platform in the east quadrant within $60 \mathrm{~s}$. Rats that could not locate the platform within $60 \mathrm{~s}$ were guided by hand to the platform and kept there for $15 \mathrm{~s}$. Platform finding time (PFT) was recorded for each $\mathrm{d} /$ starting quadrant. On $\mathrm{d} 5$ of the experiment, the hidden platform in the east quadrant was removed, and then all rats were dropped into the water in the west quadrant and were kept in the water for $60 \mathrm{~s}$, and time (s) spent in the east quadrant (which previously contained the platform) was recorded.

\section{Statistical analysis}

Data were analysed using SPSS v.11.5 for Windows (SPSS, Inc., Chicago IL.). The normality of the distribution of continuous variables was determined via the Shapiro-Wilk test. Differences between measurements on $\mathrm{d} 1,2,3$, and 4 in each group were determined using variance analysis, which was also used to identify inter-group differences, Levene's test was used to determine the homogeneity of variances. When variance was homogenous, oneway ANOVA was used, and the Welch test was used when it was not homogenous. One-way ANOVA and the Welch tests were also used to analyse inter-group differences in time spent in the east quadrant on d 5. Tukey's and Games-Howell tests were used for paired comparisons. The paired samples t test was used to identify differences between left and right hemisphere apoptotic neuron counts within each group. Inter-group differences between right and left hemisphere measurements were determined via one-way 


\section{0-733}

ANOVA and the Welch test. Descriptive statistics are shown as the mean $\pm \mathrm{SD}$. The level of statistical significance was set at $\mathrm{p}<0.05$.

\section{Results}

Overall, 2 of the 64 rats included in the study died during administration of hypoxia ( 1 from the control group and 1 from the LEV400 group). In total, 32 rats were euthanized and decapitated for assessment of neuronal apoptosis. The remaining 30 rats underwent MWM testing at age 14 weeks ( 8 in the sham group, 7 in the control group, 7 in the LEV400 group, and 8 in the LEV800 group).

\section{Neuronal apoptosis}

There were significantly more apoptotic neurons in the right hemispheres than in the left hemispheres in the control group, LEV400, and LEV800 groups ( $\mathrm{p}<0.001, \mathrm{p}<0.001$, and $\mathrm{p}<$ 0.001 , respectively). The number of apoptotic neurons in the right hemispheres in the sham, LEV400, and LEV800 groups was significantly lower than in the control group $(\mathrm{p}<0.001$, $\mathrm{p}<0.001$, and $\mathrm{p}<0.001$, respectively). The right hemisphere apoptotic neuron count in the sham group was significantly lower than in the LEV400 and LEV800 groups ( $<0.001$ and $p<0.003$, respectively). Furthermore, the right hemisphere apoptotic neuron count in the LEV800 group was significantly lower than in the LEV400 group $(\mathrm{p}=0.001)$. The left hemisphere apoptotic neuron count in the sham group, LEV400 group, and LEV800 group was significantly lower than in the control group $(\mathrm{p}<0.001, \mathrm{p}=0.001$ and $\mathrm{p}<0.001$ respectively). The left hemisphere apoptotic neuron count did not differ significantly between the sham, LEV400, and LEV800 groups $(p=0.377, p=1.000$, and $p=0.613$, respectively).

\section{MWM test}

There weren't any significant differences in PFT on $\mathrm{d} 1$ between the 4 groups $(p>0.05)$. On d 2, PFT was significantly shorter in the sham and LEV800 groups than in the control group ( $\mathrm{p}<$ 0.001 and $p=0.013$, respectively). PFT on $\mathrm{d} 3$ and $\mathrm{d} 4$ was significantly shorter in the sham and LEV800 groups than in the control group. PFT was significantly shorter on $\mathrm{d} 4$ than on $\mathrm{d} 1$ in the sham and LEV800 groups ( $p=0.001$ and $p=0.006$, respectively), but not in the control group or LEV400 group $(p=0.091$ and $p=0.096$, respectively). Mean time spent in the east quadrant on $\mathrm{d} 5$ was $17.3 \pm 6.7 \mathrm{~s}$ in the control group, versus $26.0 \pm 5.9 \mathrm{~s}$ in the sham group, $20.8 \pm 9.7 \mathrm{~s}$ in the LEV800 group, and $19.9 \pm 9.1 \mathrm{~s}$ in the LEV400 group. There weren't any significant differences in time spent in the east quadrant on $\mathrm{d} 5$ between the 4 groups $(\mathrm{p}>0.05)$.

\section{Discussion}

Seizures are not the only clinical symptom in new-borns with HIE, but such seizures exacerbate existing brain damage (18). Efficient treatment of seizures is therefore important in new-borns with HIE (19). Although LEV is not yet approved for the treatment of seizures in new-borns, it is frequently used as such off-label (20). In contrast to conventional AEDs, LEV is reportedly not neurotoxic, making it an attractive option for clinicians $(12,21)$.
Manthey et al (21) reported that LEV was not neurotoxic when administered via the intraperitoneal route to 7 -d-old rats in similar therapeutic doses in humans $\left(5,10,25,50\right.$, and $\left.100 \mathrm{mg} \cdot \mathrm{kg}^{-1}\right)$. Kim et al (12) reported that they didn't observe neuronal apoptosis associated with LEV administered via the intraperitoneal route to 7-d-old rats at higher than therapeutic doses $(250,1000$, and 1500 $\left.\mathrm{mg} \cdot \mathrm{kg}^{-1}\right)$. Similarly, in the present study, there wasn't an increase in neuronal apoptosis in response to high-dose LEV (400 and 800 $\left.\mathrm{mg} \cdot \mathrm{kg}^{-1}\right)$. In addition to the lack of neurotoxicity, LEV is an attractive treatment option because of its reported neuroprotective effects (22). Hanin and Klitgaard (11) showed that intraperitoneal administration of LEV in an adult rat model of middle cerebral artery occlusion reduced infarct volume in a dose-dependent manner. Zou et al (23) administered LEV (50 mg. $\left.\times \mathrm{kg}^{-1} \times \mathrm{d}^{-1}\right)$ intraperitoneally for $20 \mathrm{~d}$ to adult rats with traumatic brain injury beginning on $\mathrm{d} 1$ of injury and reported a significant reduction in brain damage. Talos et al (24) showed that pre-treatment with LEV (25 and 50 $\mathrm{mg} \cdot \mathrm{kg}^{-1}$ ) in 10-d-old rats with hypoxia-induced seizures reduced the cumulative duration of behavioural and electrographic seizures. Moreover, Talos et al (24) reported a significant reduction in the duration of seizures and in neuronal loss in 40-d-old rats with kainic acid-induced seizures that were pre-treated with LEV, as compared to rats not pre-treated with LEV. Despite a different study design, the present findings are in agreement with those of earlier studies. In the present study, intraperitoneal LEV administration resulted in a significant decrease in neuronal apoptosis and significant improvement in cognitive test findings.

Following reports of the neuroprotective effects of LEV and several subsequent experimental studies, many researchers have begun to wonder if LEV could be useful in treating neonatal HIBI (13-15). Kilicdag et al (13) administered intraperitoneal LEV 80 $\mathrm{mg} \cdot \mathrm{kg}^{-1}$ to 7 -d-old rats following hypoxia and continued administering LEV $40 \mathrm{mg} \times \mathrm{kg}^{-1} \times \mathrm{d}^{-1}$ for $7 \mathrm{~d}$, and reported a significant decrease in neuronal apoptosis. Similarly, Komur et al. (14) administered a single dose of LEV intraperitoneally (100 or $200 \mathrm{mg} \cdot \mathrm{kg}^{-1}$ ) after a hypoxic period in 7-d-old rats with HIBI, and observed that LEV significantly decreased neuronal apoptosis in a dose-dependent manner and significantly improved cognitive function. In contrast, Griesmaier et al (15) did not observe that LEV was neuroprotective in a neonatal rat model of HIBI, but noted that LEV further exacerbated brain damage under normothermic conditions.

In the present study, higher doses of LEV than previously studied (400 and $800 \mathrm{mg} \cdot \mathrm{kg}^{-1}$ ) were administered in a neonatal rat model of HIBI, based on earlier reports of a dose-dependent neuroprotective effect (14) and because numerous animal studies did not report toxic effects at LEV dose of 1000-1500 mg.kg ${ }^{-1}$ (12). As reported by Kilicdag et al (13) and Komur et al (14), LEV was observed to be neuroprotective in the present study's neonatal rats with HIBI. In the present study, LEV significantly decreased neuronal apoptosis and resulted in a significant improvement in cognitive function. In addition, the neuroprotective effect of LEV was higher at $800 \mathrm{mg} \cdot \mathrm{kg}^{-1}$ than at $400 \mathrm{mg} \cdot \mathrm{kg}^{-1}$. Nonetheless, the present findings are not in agreement with those of Griesmaier et al. (15), which might be because of differences in the LEV dosage and the timing of administration. In the present study, LEV was 
administered immediately after hypoxia, whereas Griesmaier et al initiated LEV treatment $2 \mathrm{~h}$ after hypoxia. In addition, in the present study a single high dose of LEV (400 or $800 \mathrm{mg} \cdot \mathrm{kg}^{-1}$ ) was administered, whereas Griesmaier et al administered a low dose of LEV (50 mg. $\mathrm{kg}^{-1}$ b.i.d.) for $3 \mathrm{~d}$.

The present study's limitation is that the mechanism of action of LEV's observed neuroprotective effect was not discerned; however, earlier studies suggested that LEV's neuroprotective effect is via various pathways $(13,14,25)$. In experimental studies, LEV prevented neonatal seizures and neuronal loss associated with these seizures (24). One of the mechanisms underlying LEV's neuroprotective effect could be its antiepileptic activity. In addition, several studies suggested that LEV inhibited glutamate transmission (26), exhibited anti-inflammatory activity (23), inhibited production of free radicals (14), and increased antioxidant enzyme levels (14, 27). LEV's neuroprotective effect observed in the present neonatal rat model of HIBI might have been via $\geq 1$ of these mechanisms.

\section{Conclusion}

The present findings indicate that LEV is neuroprotective in neonatal rats with HIBI. Based on these findings, we think that LEV could be useful in the treatment of neonatal HIBI; however, additional research is needed to further clarify the neuroprotective effect of LEV and to discern its mechanism/s of action.

\section{References}

1. Eunson P. The long-term health, social, and financial burden of hypoxic-ischaemic encephalopathy. Dev Med Child Neurol 2015; 57: 48-50.

2. Ambalavanan N, Carlo WA. Encephalopathies. In: Kliegman RM, Stanton BF, St. Geme GW, Schor NF, Behrman RE (Ed). Nelson textbook of pediatrics. 19th ed. Philadelphia: Saunders; 2011. p. 569-573.

3. Pappas A, Shankaran S, McDonald SA, Vohr BR, Hintz SR, Ehrenkranz RA et al. Hypothermia Extended Follow-up Subcommittee of the Eunice Kennedy Shriver NICHD Neonatal Research Network. Cognitive outcomes after neonatal encephalopathy. Pediatrics 2015; 135: e624-34.

4. Douglas-Escobar M, Weiss MD. Hypoxic-ischemic encephalopathy: a review for the clinician. JAMA Pediatr 2015; 169: 397-403.

5. Shetty J. Neonatal seizures in hypoxic-ischaemic encephalopathy risks and benefits of anticonvulsant therapy. Dev Med Child Neurol 2015; 57 : $40-43$.

6. Jacobs SE, Berg M, Hunt R, Tarnow-Mordi WO, Inder TE, Davis PG. Cooling for newborns with hypoxic ischaemic encephalopathy. Cochrane Database Syst Rev. 2013 Jan 31; 1: CD003311. doi: 10.1002/14651858.

7. Mruk AL, Garlitz KL, Leung NR. Levetiracetam in neonatal seizures: a review. J Pediatr Pharmacol Ther 2015; 20: 76-89.

8. Ikonomidou C. Triggers of apoptosis in the immature brain. Brain Dev 2009; 31: 488-492.

9. Ikonomidou C, Turski L. Antiepileptic drugs and brain development. Epilepsy Res 2010; 88: 11-22.

10. Mazarati AM, Baldwin R, Klitgaard H, Matagne A, Wasterlain CG. Anticonvulsant effects of levetiracetam and levetiracetam-diazepam combinations in experimental status epilepticus. Epilepsy Res 2004; 58: 167-174.
11. Hanon E, Klitgaard H. Neuroprotective properties of the novel antiepileptic drug levetiracetam in the rat middle cerebral artery occlusion model of focal cerebral ischemia. Seizure 2001; 10: 287-293.

12. Kim JS, Kondratyev A, Tomita Y, Gale K. Neurodevelopmental impact of antiepileptic drugs and seizures in the immature brain. Epilepsia 2007; 48: 19-26.

13. Kilicdag H, Daglıglu K, Erdogan S, Guzel A, Sencar L, Polat S et al. The effect of levetiracetam on neuronal apoptosis in neonatal rat model of hypoxic ischemic brain injury. Early Hum Dev 2013; 89: 355-360.

14. Komur M, Okuyaz C, Celik Y, Resitoglu B, Polat A, Balci S et al. Neuroprotective effect of levetiracetam on hypoxic ischemic brain injury in neonatal rats. Childs Nerv Syst 2014; 30: 1001-1009.

15. Griesmaier E, Stock K, Medek K, Stanika RI, Obermair GJ, Posod A et al. Levetiracetam increases neonatal hypoxic-ischemic brain injury under normothermic, but not hypothermic conditions. Brain Res 2014; 1556: 10-18.

16. Rice JE 3rd, Vannucci RC, Brierley JB. The influence of immaturity on hypoxic ischemic brain damage in the rat. Ann Neurol 1981; 9: 131-141.

17. Vorhees CV, Williams MT. Morris water maze: procedures for assessing spatial and related forms of learning and memory. Nat Protoc 2006; 1: 848-858.

18. Miller SP, Weiss J, Barnwell A, Ferriero DM, Latal-Hajnal B, Ferrer-Rogers A et al. Seizure-associated brain injury in term newborns with perinatal asphyxia. Neurology 2002; 58: 542-548.

19. Kelen D, Robertson NJ. Experimental treatments for hypoxic ischaemic encephalopathy. Early Hum Dev. 2010; 86: 369-377.

20. Silverstein FS, Ferriero DM. Off-label use of antiepileptic drugs for the treatment of neonatal seizures. Pediatr Neurol 2008; 39: 77-79.

21. Manthey D, Asimiadou S, Stefovska V, Kaindl AM, Fassbender J, Ikonomidou C, et al. Sulthiame but not levetiracetam exerts neurotoxic effect in the developing rat brain. Exp Neurol 2005; 193: 497-503.

22. Wang H, Gao J, Lassiter TF, McDonagh DL, Sheng H, Warner DS, et al. Levetiracetam is neuroprotective in murine models of closed head injury and subarachnoid hemorrhage. Neurocrit Care 2006; 5: 71-78.

23. Zou H, Brayer SW, Hurwitz M, Niyonkuru C, Fowler LE, Wagner AK. Neuroprotective, neuroplastic, and neurobehavioral effects of daily treatment with levetiracetam in experimental traumatic brain injury. Neurorehabil Neural Repair 2013; 27: 878-888.

24. Talos DM, Chang M, Kosaras B, Fitzgerald E, Murphy A, Folkerth RD, et al. Antiepileptic effects of levetiracetam in a rodent neonatal seizure model. Pediatr Res 2013; 73: 24-30.

25. Shetty AK. Prospects of levetiracetam as a neuroprotective drug against status epilepticus, traumatic brain injury, and stroke. Front Neurol 2013; 4: 172 .

26. Lee CY, Chen CC, Liou HH. Levetiracetam inhibits glutamate transmission through presynaptic $\mathrm{P} / \mathrm{Q}$-type calcium channels on the granule cells of the dentate gyrus. Br J Pharmacol 2009; 158: 1753-1762.

27. Ueda Y, Doi T, Takaki M, Nagatomo K, Nakajima A, Willmore LJ. Levetiracetam enhances endogenous antioxidant in the hippocampus of rats: in vivo evaluation by brain microdialysis combined with ESR spectroscopy. Brain Res 2009; 1266: 1-7.

Received August 3, 2016. Accepted August 12, 2016. 